\title{
Contribuciones de enfermería pediátrica en la atención de la persona adolescente en la etapa intermedia ${ }^{1}$
}

Sophía González Zúñiga ${ }^{2}$ Ana María Quezada Ugalde ${ }^{3}$

Institución: Maestría en Enfermería Pediátrica, Universidad de Costa Rica.

\section{COMO CITAR}

González, S. y Quezada, A.M. (2016). Contribuciones de enfermería pediátrica en la atención de la persona adolescente en la etapa intermedia. Rev. Enfermería Actual en Costa Rica, 31, 1-19. DOI:http://dx.doi.org/10.15517/revenf.v0i31.24573

Introducción. Se describe el acompañamiento a la persona adolescente en etapa intermedia a través de un programa de enfermería pediátrica en una institución de enseñanza media durante el año 2014, cuyo objetivo general fue articular la necesidad de conocimiento sobre sexualidad segura y prevención del consumo de drogas. Existe una necesidad imperante de mejorar la cobertura de atención debido a que el primer nivel de atención constituye la base del sistema de salud y es considerado como el espacio ideal para llevar a cabo la intervención con la población adolescente.

Método. El programa se desarrolló desde la metodología constructivista y participativa, mediante las estrategias "Entrevista de la persona adolescente", para el abordaje individual, y "talleres", para el grupal.

Resultado. A lo largo del programa se encontró una participación activa por parte de la población; el tema que requirió de mayor abordaje por parte de Enfermería Pediátrica fue el de hábitos de alimentación. Cada actividad partió del conocimiento básico que poseían los adolescentes, a quienes el programa les pareció interesante y superó sus expectativas.

Conclusión. Se concluye que las líneas de acción planteadas, individual y grupal, muestran diferentes aspectos de la adolescencia necesarios para obtener una visión completa de la persona y, por ende, ofrecer un abordaje más acertado; además de que el criterio del profesional de enfermería pediátrica es indispensable para responder a las necesidades identificadas y la caracterización de factores de riesgo y/o protectores.

Palabras clave: adolescencia, colegio, enfermería-pediátrica, programa-intervención.

\footnotetext{
${ }^{1}$ Fecha de recepción: 18 de junio del 2015

Fecha de aceptación: 14 marzo del 2016

${ }^{2}$ Enfermera. Máster en Enfermería Pediátrica, Coordinadora Curso de Formación ATAPS, Escuela de Enfermería. Universidad de Costa Rica. Correo electrónico: sophiawwjd@ gmail.com

${ }^{3}$ Enfermera. Máster en Enfermería Pediátrica, Docente, Escuela de Enfermería. Universidad de Costa Rica. Correo electrónico: ana.QUEZADAUGALDE@ucr.ac.cr
} 


\title{
Contributions in pediatric nursing care teenager person in middle stage ${ }^{1}$
}

\author{
Sophía González Zúñiga ${ }^{2}$ \\ Ana María Quezada Ugalde ${ }^{3}$
}

Institution: Masters degree in Pediatric Nursing, University of Costa Rica.

\section{CITED}

González, S. y Quezada, A.M. (2016). Contributions in pediatric nursing care teenager person in middle stage. Rev. Enfermería Actual en Costa Rica, 31, 1-19. DOI:http://dx.doi.org/10.15517/revenf.v0i31.24573

\begin{abstract}
Introduction. Accompaniment to the adolescent described in intermediate stage through a program of Pediatric Nursing in an institution of secondary education in 2014, whose main objective was to articulate the need for knowledge about safe sex and prevention of drug use. There is an urgent need to improve care coverage because the first level of care is the foundation of the health system and is considered as the ideal space to carry out the intervention with the adolescent population.

Method. The program was developed under the constructivist and participative methodology, using strategies: "Interview of the adolescent" for individual approach and "workshops" for the group approach.

Result. Throughout the program active participation by the population, was found the situation required greater approach by Pediatric Nursing in eating habits; in each activity we started from the basic knowledge they possessed adolescents, the program seemed interesting and exceeded their expectations.

Conclusion. It was concluded that the lines of action raised, individual and group showed different aspects of adolescence needed to get a complete picture of the person and thus provide a more accurate approach; the criterion of professional pediatric nursing is essential to meet the identified needs and characterization of risk factors and / or protective.
\end{abstract}

Key words: adolescence, high-school, intervention-program, pediatric-nursing.

\footnotetext{
${ }^{1}$ Date of receipt: June 18, 2015

Date of acceptance: March 14, 2016

${ }^{2}$ Nurse. Master degree in Pediatric Nursing. Coordinator Training Course of ATAPS, School of Nursing. University of Costa Rica. Costa Rica. E-mail: sophiawwjd@gmail.com

${ }^{3}$ Nurse. Master degree in Pediatric Nursing. Professor of School of Nursing. University of Costa Rica. Costa Rica. E-mail: ana.QUEZADAUGALDE@ucr.ac.cr
} 


\section{Contribuições da enfermagem pediátrica no atendimento da pessoa adolescente na etapa intermediária ${ }^{1}$}

Sophía González Zúñiga ${ }^{2}$ Ana María Quezada Ugalde ${ }^{3}$

Instituição: Mestre em Enfermagem Pediátrica, Universidade da Costa Rica.

\section{CITAÇÃO}

González, S. y Quezada, A.M. (2016). Contribuições da enfermagem pediátrica no atendimento da pessoa adolescente na etapa intermediária. Rev. Enfermería Actual en Costa Rica, 31, 1-19. DOI: http://dx.doi.org/10.15517/revenf.v0i31.24573

\section{RESUMO}

Introdução. Descreve-se o acompanhamento da pessoa adolescente na etapa intermediária através de um programa de enfermagem pediátrica em uma instituição de ensino médio durante o ano 2014, cujo objetivo geral foi articular a necessidade de conhecimento sobre sexualidade segura e prevenção do consumo de drogas. Existe uma necessidade imperante de melhorar a cobertura de atendimento, devido a que o primeiro nível de atenção constitui a base do sistema de saúde e é considerado como o espaço ideal para realizar a intervenção com a população adolescente.

Método. O programa se desenvolveu desde a metodologia construtivista e participativa, mediante as estratégias "Entrevista da pessoa adolescente", para a abordagem individual, e "oficinas", para o grupo.

Resultado. Ao longo do programa se encontrou uma participação ativa por parte da população; o tema que requeriu maior abordagem por parte da Enfermagem Pediátrica foi o dos hábitos de alimentação. Cada atividade partiu do conhecimento básico que tinham os adolescentes, para os quais o programa pareceu interessante e superou suas expectativas.

Conclusão. Conclui-se que as linhas de ação planteadas, individual e grupal, mostram diferentes aspectos da adolescência necessários para obter uma visão completa da pessoa e, com isso, oferecer uma abordagem mais acertada; além de que o critério do profissional de enfermagem pediátrica é indispensável para responder às necessidades identificadas e a caracterização de fatores de risco e/ou protetores.

Palavras-chave: adolescência, colégio, enfermagem-pediátrica, programa-intervenção.

\footnotetext{
${ }^{1}$ Data de recepção: 18 de junho de 2015

Data de aceitação: 14 de março de 2016

${ }^{2}$ Enfermeira. Mestre em Enfermagem Pediátrica. Coordenador de Curso de Formação ATAPS, Escola de Enfermagem. Universidade da Costa Rica. Correio eletrônico: sophiawwjd@ gmail.com

${ }^{3}$ Enfermeira. Mestre em Enfermagem Pediátrica. Professor Escola de Enfermagem, Universidade da Costa Rica. Correio eletrônico: ana.QUEZADAUGALDE@ucr.ac.cr
} 


\section{Revista Electrónica Enfermeria Actual en costa Rica}

\section{www.revenf.ucr.ac.cr}

\section{INTRODUCCIÓN}

La población adolescente es protegida a través de un marco jurídico básico, tanto a nivel internacional, con la Declaración de Ginebra sobre los Derechos del Niño del año 1924 y la Convención sobre los Derechos del Niño de 1989 (Organización de Naciones Unidas, 1989), como a nivel nacional, con el Código de la Niñez y la Adolescencia en 1998 y la Política Nacional para la Niñez y Adolescencia 2009- 2021 (PANI- UNICEF, 2009). Estas políticas tienen como fin defender los derechos de todos los y las adolescentes.

En torno al tema, el Fondo de las Naciones Unidas para la Infancia (2011) determina que la cantidad de adolescentes a nivel mundial ha venido en aumento hasta alcanzar un 19\% de la población; por su lado, en Costa Rica, el Instituto Nacional de Estadísticas y Censos (2011) indica que, del total de costarricenses, el 18,4\% representa a adolescentes entre los diez y los diecinueve años.

En el ámbito de la salud, uno de los grupos etarios de menor asistencia a los servicios y, por tanto, de menor cobertura, es la población adolescente (Caja Costarricense de Seguro Social, 2012) que por sus características de vulnerabilidad propias de la adolescencia construcción de identidad, autoconcepto, toma de decisiones, cambios físico-emocionales, entre otras, justifica la relevancia de brindarles un cuidado especializado.

Debido a que el sistema nacional de salud opera en tres niveles de atención dentro de los cuales enfermería se encuentra presente, se considera el primer nivel de atención, cuya proyección es la comunidad, por consiguiente $\mathrm{y}$, por consiguiente, la base del sistema, como el espacio ideal para llevar a cabo la intervención con la población adolescente. Dado lo anterior, para efectos de este programa la atención de enfermería pediátrica se desarrolló dentro del escenario educativo con la intención de obtener un mayor impacto, accesibilidad, acompañamiento y seguimiento, centrado en la valoración del crecimiento y desarrollo del individuo, una herramienta clara y efectiva que facilitó la labor a través de acciones de promoción de la salud y prevención de la enfermedad.

Ante la evidencia descrita, este artículo corresponde a la planificación, implementación y resultados de un programa de enfermería pediátrica para la atención a la persona adolescente en etapa intermedia, el cual es producto de una fase diagnóstica y se enmarca dentro de un proceso de investigación acción. El objetivo general de dicho programa fue el de articular la necesidad de conocimiento sobre sexualidad segura y prevención del consumo de drogas con una propuesta de atención por parte de enfermería pediátrica.

\section{MATERIALES Y MÉTODOS}

La población estuvo formada por las 684 personas adolescentes inscritas como estudiantes de octavo y noveno año de secundaria, durante el año 2014 (González-Quirós, 2010; Retana-Goñi, 2007). De acuerdo con la capacidad operativa de recolección y análisis con la que contaron las investigadoras, y en relación con los recursos de tiempo y espacio disponibles, se determinó una muestra de tipo no probabilística, con un total de 32 personas adolescentes estudiantes, durante el año 2014. Los criterios para la escogencia de fueron ser estudiante regular del colegio en los niveles de octavo y noveno año, tener anuencia a participar en el estudio y pertenecer a la etapa intermedia de la adolescencia. 


\section{Revista Electrónica Enfermeria Actual en costa Rica}

El programa de Enfermería Pediátrica para la atención de la persona adolescente estuvo inmerso dentro de un proceso constructivista y participativo, en el cual las investigadoras desempeñaron un rol de facilitadoras, mientras que los adolescentes construyeron el conocimiento a través de sus perspectivas, experiencias, y participación activa (Krause, 1995). Debido a la relevancia de las experiencias subjetivas de la población adolescente, su comprensión y el entorno en las que tienen lugar, es posible afirmar que se trató de un diseño de investigación-acción (Vidal-Ledo y Rivera-Michelena, 2007); por lo tanto, a lo largo del proceso fue posible investigar e intervenir al mismo tiempo.

El lugar donde se implementó el programa fue el Liceo Samuel Sáenz Flores, ubicado en la provincia de Heredia, Costa Rica, y constó de por 4 fases, a saber:

1. Etapa diagnóstica: se diagnostica el problema, lo cual demandó conocer a fondo la naturaleza de la población adolescente mediante una inmersión completa de las investigadoras en su contexto. Para el desarrollo de la inmersión inicial se llevó a cabo una observación durante las actividades realizadas en la semana cívica. Además, se planeó grupos focales con los adolescentes para que señalaran las necesidades que cursan durante su adolescencia intermedia y aquellas estrategias que deseaban utilizar para su abordaje y modificación.

En esta etapa se realizó el primer proceso de triangulación, que radicó en una comparación entre las realidades de los adolescentes obtenidas por las diferentes técnicas de recolección de datos, y el referente teórico acerca de lo que es su adolescencia intermedia.

2. Diseño del programa: una vez culminado el diagnóstico se procedió a la elaboración del programa de enfermería pediátrica para la atención de la persona adolescente que pretendió dar respuesta a los hallazgos. Dicho programa constó de dos estrategias de abordaje, individual y grupal, las cuales se desarrollaron de manera simultánea.

La atención individual del programa se basó en la técnica de la entrevista de la persona adolescente, mediante el uso de la "Guía para la entrevista clínica de la persona adolescente", construida por las investigadoras tomando como base el Sistema Informático del Adolescente propuesto por Belamendia et al. (2010). Como parte de esta, se realizó una valoración antropométrica con el fin de determinar el estado nutricional de los participantes, según la gráfica del Índice de Masa Corporal (IMC) para hombres o mujeres de 5 a 19 años, elaborada por la Caja Costarricense del Seguro Social (CCSS).

Una vez finalizada cada una de las entrevistas, se procedió a realizar una categorización respecto del riesgo -existente o no- para la persona adolescente, mediante el algoritmo de riesgos y acciones confeccionado a partir de la revisión de la Integración del Manejo del Adolescente y sus necesidades (IMAN) de la Organización Panamericana de la Salud (2010). Dicho algoritmo incluyó dimensiones como residencia, alimentación, rendimiento académico, percepción de sí mismo, hábitos, sexualidad y consumo de drogas, por lo que la presencia de dos o más condiciones de riesgo en las diferentes dimensiones que incluye el algoritmo, cataloga a la persona adolescente en bajo o alto riesgo, por lo que la existencia de una sola corresponde a la clasificación de "sin riesgo aparente". 


\section{Revista Electrónica Enfermeria Actual en costa Rica}

En caso de encontrar alguna situación que requiriera de atención especializada se contó con un sistema de referencia hacia instituciones como el Instituto sobre Alcoholismo y Farmacodependencia (IAFA) y la CCSS (áreas de salud y Clínica del Adolescente).

Como último elemento, se elaboró un reporte de los hallazgos más relevantes de la entrevista dirigida a la persona encargada de cada menor, con el fin de hacer constar su participación previamente autorizada.

Para efectos del abordaje a nivel grupal se buscó enfatizar a las temáticas desexualidad segura y prevención del consumo de drogas en los adolescentes, que correspondieron a las prioridades exteriorizadas por ellos. La modalidad seleccionada para la implementación de los temas fue la de taller, debido a su flexibilidad, apropiación y el desarrollo de conocimientos, actitudes y competencias, de manera que sea el sujeto de investigación quien construya el conocimiento $\underline{\text { (Ghiso, 1999). }}$

3. Implementación del programa: La tercera etapa correspondió a poner en marcha el programa, durante el cual se trabajó en conjunto con la población de adolescentes; al desarrollar las estrategias individual y grupal se cumplió con dos funciones específicas: evaluar los avances y recoger nuevamente la "viva voz", opiniones, experiencias y sentimientos de los participantes.

A lo largo de la implementación del programa de enfermería pediátrica para la atención de la persona adolescente, hubo un segundo proceso de triangulación que involucró tanto a la población meta, las investigadoras y el referente teórico, con el fin de obtener una retroalimentación constante que evidenció el logro de los objetivos.

4. Evaluación del programa: Al final de la implementación del programa, se analizó nuevamente los datos recolectados, entre ellos las experiencias de los adolescentes, los nuevos aprendizajes, las modificaciones o mejoras en sus conductas, entre otros; lo anterior condujo a un "ciclo de retroalimentación" que implicó adecuarse a las contingencias surgidas a lo largo del proceso y que buscó solucionar el problema de estudio.

Los instrumentos utilizados para evaluar el programa fueron: la hoja de vivencias, para el trabajo individual, y preguntas generadoras para la estrategia grupal. La primera captó las reacciones, actitudes y expresiones tanto de los participantes, como de las investigadoras a lo largo de la entrevista, además de registró la clasificación del riesgo según algoritmo y el abordaje que se brindó a las situaciones encontradas. Las preguntas se enfocaron en las expectativas, el interés generado y recomendaciones planteadas por los participantes.

\section{CONSIDERACIONES ÉTICAS}

En cuanto a los principios bioéticos, En el proceso investigativo se consideró los de beneficencia, no maleficencia, autonomía y justicia, lo cual motivó el diseño del consentimiento informado en el que la persona encargada autoriza al estudiante a participar en el estudio. Asimismo, se utilizó un asentimiento informado dirigido a la persona adolescente para registrar su anuencia a-formar parte de la investigación. 


\section{Revista Electrónica Enfermeria Actual en costa Rica}

\section{www.revenf.ucr.ac.cr}

\section{RESULTADOS}

Para la estrategia del abordaje individual, se contó con la guía para la entrevista de la persona adolescente que incluyó los siguientes puntos clave: datos socio-demográficos, familia (con la construcción del genograma), alimentación, actividad física, patrón de sueño, eliminaciones, padecimiento de alguna enfermedad y su tratamiento, rendimiento académico, relaciones interpersonales, relaciones de pareja, sexualidad, consumo de sustancias y percepción de sí.

En total se efectuó 22 entrevistas: 9 respondidas por mujeres y 13, por hombres. La duración de cada entrevista fue de aproximadamente 45 minutos. El espacio seleccionado para su implementación fue el comedor del colegio mientras este no estaba siendo utilizado. La dinámica de trabajo tuvo como particularidad que una de las investigadoras llevó a cabo la entrevista y el abordaje de la persona, mientras que la otra cumplió un rol de observadora del proceso.

La situación que requirió con mayor frecuencia el abordaje de enfermería pediátrica fue la de hábitos de alimentación puesto que se presentaron rasgos como el consumo elevado de comida "chatarra" con una frecuencia alrededor de 4 a 5 veces por semana. El poco consumo de frutas en la dieta de la persona adolescente corresponde a otra característica hallada, lo cual se pudo observar en casos en los que el consumo es semanal o de cada tres días. También se mostró la tendencia a no cumplir con los cinco tiempos de alimentación recomendados que, en su mayoría, se reduce a tres tiempos y no necesariamente en los horarios esperados; sin embargo, al menos uno de ellos se realiza en compañía del grupo familiar. En general se presentó un patrón de consumo de agua diario y con raciones entre los 4-8 vasos.

En cuanto a la valoración física que incluyó antropometría, toma de presión arterial, y -en caso de que los lo refirieran- detección de malestares físicos, se obtuvo que los adolescentes presentan un estado nutricional sin alteraciones para su edad, a excepción de tres casos que tuvieron un estado de sobrepeso, dos de ellos obesidad y una con bajo peso, razón por la que se programó una cita de control antropométrico para determinar la necesidad de efectuar una referencia con el fin de brindar una atención especializada. Posterior a dicho control. se encontró que la mayoría de participantes se ubicaba en el estado nutricional esperado para su edad.

Dada la situación anterior, las facilitadoras realizaron recomendaciones enfocadas en el establecimiento de horarios para la alimentación, cumplimiento de tiempos de comidas, alimentos que deben incluirse según los requerimientos de la persona adolescente y la permanencia del hábito del consumo de agua.

Respecto de los hábitos de eliminación fue posible determinar que un elevado número de adolescentes defeca diariamente y sin dificultad. En las situaciones contrarias, se brindó orientación en cuanto a tener un mayor consumo de fibra y la práctica de actividad física como parte de sus hábitos de vida saludable. En relación con este último factor, se identificó que menos de la mitad de la población acostumbra realizar ejercicio: los participantes restantes no realizan ningún tipo de deporte o cumplen únicamente con las lecciones de Educación Física que imparten en el colegio. 


\section{Revista Electrónica Enfermeria Actual en costa Rica}

De acuerdo con los hallazgos relacionados con los hábitos del sueño de los participantes, se hace hincapié en que la mayoría duerme alrededor de 8 horas al día, y una tercera parte duerme 6 horas o menos, ya que se acuestan tarde y se levantan temprano para asistir al colegio. Aunado a lo anterior hubo sujetos que mencionaron dificultad para conciliar el sueño o que despertaban en varias ocasiones durante la noche.

Sobre el uso del tiempo libre, dedican tiempo a ver televisión, videojuegos, usar la computadora, salir con amistades y participar en grupos sociales, sin embargo, se observó que en todos existe la tendencia de acceder a internet y redes sociales por medio del celular a lo largo del día.

Un elemento que surgió durante la implementación de las entrevistas fue la presencia de malestares físicos en más de la mitad de ellos, dentro de los cuales se pueden citar, en orden de mayor a menor frecuencia, los siguientes: mareos, cefaleas, dolor abdominal y cervical. En el caso particular de las mujeres, sobresale la dismenorrea como su principal problema físico (8 de 9 adolescentes).

En general, consumen o han consumido algún tipo de sustancia, -drogas lícitas- y solo un caso refirió haber consumido marihuana. La sustancia más común fue el alcohol, cuyo primer contacto se basó en el ofrecimiento en el entorno familiar o entre pares, con el objetivo de "probar", no obstante, ninguno expresa que sea de uso frecuente. Además, fueron las mujeres quienes reportaron mayor contacto con dicha sustancia en relación con los hombres.

Por otro lado, se discutió temas relacionados con la sexualidad de la persona adolescente, aunque cabe destacar que durante las entrevistas no hubo gran apertura por parte de ellos para comentar acerca de estos tópicos, no obstante, se logró conocer que en su mayoría no ha dado inicio con su vida sexual y que para el momento no tienen pareja o algún tipo de relación; existió un caso que ha tenido primeros contactos físicos, pero no mantiene una relación afectiva en la actualidad; por último, se detectó 3 adolescentes que poseen un noviazgo sin haber iniciado una vida sexual.

En cuanto a la evaluación clínica del desarrollo puberal llevada a cabo con el test de tanner, es relevante mencionar que todos presentaron un desarrollo adecuado para su edad y respecto la aparición de los primeros cambios puberales.

Posterior al análisis global de la situación de cada joven, se clasificó a los participantes de acuerdo con el algoritmo de riesgos y acciones: al respecto, 14 se ubican en la categoría de "sin riesgo aparente", aunque ninguno en la de "alto riesgo". No obstante, ocho adolescentes se encuentran en una condición de "bajo riesgo", los cuales se subdividieron de acuerdo con la presencia de características en común.

La categorización de los estudiantes consideró también la valoración de factores protectores y de riesgo presentes. En el ámbito individual, se incluye una adecuada percepción de sí, crecimiento y desarrollo normal para su edad, esquema de vacunación completo, ausencia de padecimientos crónicos, presencia de relaciones entre pares dentro y fuera de la institución educativa, establecimiento de metas educacionales y metas vocacionales (tentativas), y reconocimiento de sentimientos como alegría y éxito. En lo referente al área familiar 


\section{Revista Electrónica Enfermeria Actual en costa Rica}

una buena percepción de su núcleo, relaciones cercanas con sus padres, madres y/o demás miembros, satisfacción de las necesidades básicas, apoyo a tanto a nivel emocional como académico, y contar con mayor libertad para la toma de decisiones o participación en actividades de su agrado.

Respecto a la comunidad, se encontró la existencia de un centro educativo que promueve un ambiente de oportunidades recreativas, deportivas, sociales y educacionales, un medio que procura proteger a la población y atenuar riesgos; en cuanto a los barrios donde residen no existe áreas clasificadas como comunidad urbanomarginal.

Luego de la clasificación, las facilitadoras realizaron una devolución para brindar al individuo una serie de recomendaciones u orientación. Del total de estudiantes, uno de los menores fue referido a la Clínica del Adolescente del Hospital Nacional de Niños, dado que presentaba un estado nutricional de obesidad mayor aún del que se encontraba unos meses antes.

A partir de la hoja de vivencias, se encontró que durante la entrevista los adolescentes cumplieron con un patrón de comportamiento muy similar, al inicio de cada sesión se mostraron tímidos, reservados en sus respuestas, serios, atentos, con ciertas características de lenguaje corporal tales como el no mantener un contacto visual, brazos cruzados, sin uso de ademanes, movimientos repetitivos con alguno de sus miembros, incluso mantuvieron sus pertenencias en sus regazos o espalda. Conforme avanzó la entrevista, se mostraron más confiados, espontáneos, seguros y con respuestas más detalladas, usaron gestos y ademanes, sonrieron, cambiaron de posición, realizaron preguntas con mayor libertad y dejaron a un lado sus objetos personales.

En relación con la entrevistadora, mantuvo contacto visual, demostró respeto, tornó la entrevista en una conversación dirigida por medio de preguntas abiertas o generadoras que permitieran a la persona describir ampliamente sus opiniones, sentimientos y experiencias, frente a las cuales mostró interés y empatía, además de hacer retroalimentación para aquellas áreas que necesitaron reforzarse o modificarse y devolución en varias ocasiones para cerciorarse de la información obtenida, todo lo anterior haciendo uso de un lenguaje adecuado y comprensible para los jóvenes.

Por otro lado, la estrategia de abordaje grupal se llevó a cabo a través de los talleres Sexualidad segura y Prevención de consumo de drogas. El primero de ellos requirió de dos sesiones para abarcar la población participante, mientras que el de prevención de consumo de drogas precisó de tres momentos. La metodología empleada se fundamentó en ser constructivista-participativa, la cual contó con buena aceptación por parte del grupo, puesto que los participantes comentaron haber disfrutado de los talleres, en especial por la oportunidad que se les brindó de formar parte activa en el desarrollo de estos.

El taller de sexualidad segura incluyó seis actividades educativas, más una rompe-hielo, ejecutadas en un ambiente de confidencialidad y confianza con el propósito de propiciar la participación de los adolescentes. Tuvo una duración de 1 hora y 30 minutos. 


\section{Revista Electrónica Enfermeria Actual en costa Rica}

La primera actividad del taller sobre sexualidad segura, "Caja del saber", consistió en la discusión acerca de las expresiones, estereotipos y posiciones que cada uno manejaba en torno a la temática, y su posterior clasificación en mitos o realidades; por consiguiente, brindaron sus aportes durante la discusión que surgió al clasificarlas, de manera que se esclareciera si era correcta o no la información que conocían. Desde el inicio del taller también se efectuó la actividad "Experimentando estar embarazada (o)", la cual consistió en sostener un vientre de 8 meses de gestación durante al menos 20 minutos. En cada sesión participaron una mujer y un hombre expresando sensaciones de incomodidad, afirmando que era muy pesado, que tuvieron que cambiar su manera de caminar y sentarse e, incluso, surgieron expresiones sobre el esfuerzo realizado por parte de sus madres al estar embarazadas.

Hacia el final del taller, se hizo un cierre de esta dinámica en la que convergieron los temas de embarazo en la adolescencia y proyecto de vida, con el fin de contrastar lo que planean para sus vidas y el cambio que implica un embarazo o la presencia de un bebé durante esta fase, con el propósito de empoderar a la persona adolescente en la toma responsable de decisiones y el asumir lo que estas conllevan.

Como tercera actividad se construyó un espacio de simulación donde se mostró el uso de los métodos anticonceptivos más comunes, como lo son el preservativo masculino, anticonceptivos orales e inyectables. En cuanto al manejo correcto del preservativo masculino, cada uno de los participantes tuvo la experiencia de colocarlo y de realizar una retroalimentación ante posibles fallas durante la ejecución. De igual forma, se familiarizaron con la presentación de los anticonceptivos orales e inyectables, conocieron la forma correcta para su administración de acuerdo con las características del fármaco (21 o 28 tabletas), los principales errores en su empleo, efectos secundarios y la importancia de contar con una valoración médica previa para su prescripción.

Dado que se logró crear un espacio de confianza, los adolescentes evidenciaron un interés real a lo largo de toda la actividad, fue posible evacuar sus dudas, discutir acerca de estereotipos y brindar información que les permita tomar control sobre sus decisiones y experiencias en torno a la sexualidad.

En la dinámica "Una huella para toda la vida", se estableció relaciones sentimentales entre los participantes haciendo uso de situaciones hipotéticas con el fin de no generar malentendidos. Conforme se describía las supuestas relaciones, se narró la historia que poseía cada uno de ellos o ellas en cuanto a sus anteriores vínculos afectivos y físicos. Además, se discutió que cada una de esas relaciones podría dejar una "huella" en ámbitos como el físico, (enfermedades de transmisión sexual, embarazo), emocional (tristeza, ira, alegría, inseguridad) y/o social (etiquetas), lo cual origina una cadena de efectos que envuelve a todas las personas inmersas.

Los comportamientos y reacciones frente a lo suscitado, arrojaron datos significativos. La población que asistió participó en su totalidad y demostró haber interiorizado el papel asignado, así como una preocupación por la cantidad de "huellas" que poseían, y actitudes de sorpresa, risas, ansiedad, entre otras.

Posteriormente se realizó la construcción de proyecto de vida titulado: “¿Qué quiero "ha- ser"?" Cabe mencionar que se respetó la privacidad de la información, por lo que no se discutió grupalmente; sin embargo, se 


\section{Revista Electrónica Enfermeria Actual en costa Rica}

comentó que los proyectos están enfocados principalmente en terminar sus estudios, adquirir un grado profesional, un trabajo, bienes materiales y dejan a largo plazo la formación de un núcleo familiar estable, aspecto que dio pie a la discusión citada previamente en la actividad de simulación del embarazo.

Asimismo, se utilizó la técnica de blog para informar y discutir aquellas inquietudes presentes en los y las adolescentes, por un lapso de una semana. El blog se llamó "Entre vos y yo", el manejo de este se explicó al finalizar el taller. Sin embargo, no se contó con participación de ninguno, por lo que no fue posible cumplir con su propósito. La principal razón se vinculó con la poca familiaridad del manejo de este tipo de espacios virtuales.

El segundo taller concierne al tema de prevención del consumo de drogas, el cual incluye cuatro actividades educativas, más una rompe-hielo. La primera actividad la titulada "El enganche" para ejemplificar la puerta de entrada al consumo de drogas. En esta, dos adolescentes accedieron a que sus ojos fueran cubiertos mientras se les ofreció alimentos que desconocían; el resto del grupo se dividió con el fin de que, independientemente del tipo de alimentos, unos motivaran el consumo y la otra parte les indicaran lo desagradables que eran. Algunas de las expresiones utilizadas fueron: “créame, se va a arrepentir" (TallerDrog No.1), "confíe en mi”" (TallerDrog No.3) y "dígame... ¿qué pierde con probar?” (TallerDrog No.2). A pesar de la presión que existía alrededor de ellos y ellas, la decisión final era tomada por quienes tenían sus ojos vendados que en muchos de los casos rechazaron el ofrecimiento y unos pocos se animaron a probar todo lo ofrecido.

Al finalizar la actividad, se dio un espacio para comentar la experiencia y las razones de sus decisiones. Dentro de las cuales se resaltó el temor, la confianza en la persona que ofrece, inseguridad, presión de los demás y curiosidad. Las facilitadoras cierran la dinámica estableciendo un vínculo entre lo acontecido y el fenómeno de las drogas.

Posteriormente, haciendo uso del libro: Drogas, preguntas más frecuentes (Instituto Costarricense de Drogas, s.f.), se facilitó información sobre los efectos que pueden generar las drogas y el porqué se consumen. El grupo se dividió en dos posiciones, una a favor y otra en contra del consumo de sustancias lícitas o ilícitas, con el fin de que reflexionaran acerca de la temática desde la perspectiva indicada y construyeran argumentos para defenderla e iniciar el debate. A lo largo de este, se observó la facilidad con la que se desenvolvieron quienes mantenían un criterio en contra del consumo. En sus afirmaciones resaltaron los efectos que producen las drogas y su perjuicio a nivel físico, emocional, familiar y social; además exteriorizaron las razones por las que las personas suelen consumir, entre ellas: necesidades afectivas, presión social y pares. A pesar de que pocos adolescentes cumplieron su rol de estar a favor del consumo, hubo jóvenes que expresaron no poder cumplir con esta posición puesto que no coinciden al respecto.

Una vez realizado el cierre del debate, se da pie a la actividad relacionada con el proceso de adicción, representada por medio de un tobogán. Como parte de la dinámica se brindó un rol para cada participante, a saber: nunca subir al tobogán, llegar a la primera, segunda o tercera grada, alcanzar la parte más alta, deslizarse por el tobogán y tratar de devolverse en la rampa, y motivar o disuadir a los compañeros a deslizarse. La tendencia fue que cada uno cumpliera su papel, sin embargo, hubo quienes se mostraron indecisos y frente a la presión del grupo descendieron por la rampa aún no siendo ese su rol. Durante el conversatorio, los jóvenes 


\section{Revista Electrónica Enfermeria Actual en costa Rica}

enfatizaron situaciones como la dificultad para devolverse después de deslizarse y el acceder a continuar a pesar de que no les correspondiera.

En cuanto al uso del recurso audiovisual "Saber elegir, saber ganar" (Instituto Costarricense de Drogas, s.f.), el grupo refirió que a pesar de que no es un video actual, muestra realidades vividas por la población adolescente tales como asistir a fiestas donde se distribuyen drogas, ofrecimiento por parte de compañeros o conocidos, retar a la autoridad, percibir el uso de drogas como una salida fácil, y la importancia del apoyo de la familia y los amigos.

Al finalizar cada taller se les solicitó que respondieran a las siguientes interrogantes: ¿qué esperaban de este taller?, ¿les pareció interesante, sí o no y por qué? y ¿qué le cambiarían o agregarían? En la primera de ellas, en particular para el caso del taller de sexualidad segura, manifestaron que esperaban algo más sencillo, una charla con información sobre el tema, algo aburrido, información sobre lo mismo y con pocas actividades, mientras que para el taller de prevención de consumo de drogas esperaban mayor diversión, actividades e información.

En cuanto a la percepción de si fue o no interesante, la totalidad afirma que en los dos casos sí lo fue, debido a que se les facilitó información y respuestas a las preguntas que plantearon, además de que la información fue útil y de gran ayuda para tomar las mejores decisiones.

Por último, en la tercera pregunta, en general manifestaron que no era necesario hacer cambios en los talleres, sin embargo, cabe rescatar que en el taller sobre sexualidad segura se recomendó el uso de un esquema anatómico y para el de drogas, profundizar en los efectos y tipos de drogas.

A lo largo de la implementación de los talleres, las investigadoras desempeñaron funciones como orientar los temas en discusión, delimitar la información de acuerdo con los objetivos propuestos, a las necesidades y características de la población adolescente, brindar las instrucciones para cada dinámica, dar dirección a las reacciones que surgieron (bromas o comentarios fuera del tema) y propiciar la participación de quienes se mostraron introvertidos.

\section{DISCUSIÓN}

El análisis de los datos se efectúa desde la percepción de la persona adolescente como un individuo que experimenta cambios constantes a lo largo de esta etapa de la vida, así como necesidades afectivas, físicas, y académicas particulares que se vinculan con el contexto en el que se desenvuelve, motivo por el que elementos como la familia, pares, comunidad y centro educativo tienen gran relevancia en el desarrollo de esta población y en generar esfuerzos para intervenir desde Enfermería Pediátrica.

Las estrategias seleccionadas para la intervención fueron la grupal e individual, las cuales se justifican en el hecho de que existen temáticas que se pueden desarrollar de forma colectiva, así como otras que requieren de mayor privacidad, profundidad e intimidad. Además, al utilizar ambas estrategias fue posible comparar el 


\section{Revista Electrónica Enfermeria Actual en costa Rica}

comportamiento y respuestas de los participantes, en situaciones en las que se encontraron solos, frente a espacios que compartieron con sus pares (Zubarew, 2013).

Para la entrevista de la persona adolescente, utilizada en el abordaje individual, se seleccionó el comedor como un entorno agradable y cómodo, que además contó con buena iluminación y ventilación, privacidad, un lugar no ajeno y con las condiciones aptas para realizar las diferentes actividades planificadas (valoración antropométrica, toma de presión arterial, entre otras) (Ruiz-Lázaro, 2009).

Tal como se describió en el apartado anterior, los hábitos de alimentación de entrevistados confirman lo argumentado por Marugán de Miguelsanz, Monasterio-Corral, y Pavón-Belinchón (2010), quienes señalan que es común en la población adolescente comer fuera de casa, aumentar el consumo de comida hipercalórica, omitir tiempos alimentarios, imponerse dietas, entre otros aspectos, que los posiciona en una época de alto riesgo nutricional. En relación con lo mencionado, el Grupo AVENA (2003), indica también que dentro de los hábitos alimentarios de los adolescentes se encuentra el de no desayunar, consumir pequeñas ingestas frecuentes entre comidas principales y de alto valor calórico. Cabe destacar que el tiempo de comida que la población adolescente refirió compartir con su sistema familiar fue considerado un factor de protección relativo a la familia puesto que es un momento en el que se dedican tiempo (Zubarew, 2013).

En relación con la actividad física en la persona adolescente, la Federación Española de Medicina del Deporte (2008), recomienda que esta población practique 60 minutos diarios de actividad moderada o algo menos si la intensidad es mayor, situación que no se observó en la mayoría de los casos entrevistados.

El estado nutricional de los mostró una estrecha relación con el estilo de vida que presentan, así por ejemplo elementos como una alimentación acorde con sus necesidades, más la práctica de actividad física y aspectos propios de la etapa de crecimiento, reflejan un estado nutricional normal según la gráfica de IMC. Por su parte, quienes presentaron un estado nutricional alterado se vincularon con hábitos inadecuados.

Otro de los hábitos en discusión, es la cantidad y calidad del sueño en los adolescentes: al respecto, PinArboledas (2010) establece que entre 8 -9 horas de sueño son las necesarias para esta población con el propósito de favorecer los niveles de atención, concentración, razonamiento y habilidades psicomotoras, así como en la segregación de la hormona de crecimiento. Respecto de la dificultad para conciliar el sueño o despertares nocturnos, Quevedo-Blasco y Quevedo-Blasco (2011), perciben como alteraciones de la calidad del sueño en la adolescencia la presencia de los despertares nocturnos, la existencia y duración de siestas, el tiempo y la manera de conciliar el sueño.

Con base en los datos obtenidos sobre el uso del tiempo libre, se evidencia lo planteado por Echeburúa (2010), quien menciona que el frecuente uso de las nuevas tecnologías de la información y comunicación (TIC) entre la población joven se ha convertido en una realidad cotidiana. El mismo autor expone que las TIC simplifican considerablemente los quehaceres de la vida diaria, cuyo atractivo para los adolescentes radica en la interactividad, respuesta rápida, y las múltiples experiencias recreativas. El grupo participante no se ubica en la categoría de abuso de las TIC, ya que no descuidan el resto de sus actividades diarias, mas al encontrar la 


\section{Revista Electrónica Enfermeria Actual en costa Rica}

conducta de permanecer conectados por tantas horas, las facilitadoras insistieron en que participaran en las tareas del hogar, dedicaran tiempo a realizar ejercicio, u otras actividades de índole social.

Con respecto a los malestares físicos hallados, esta realidad es congruente con lo que exponen LlusentGuillamet, Agüero-Martínez, y Cornellà-Canals (2007), quienes describen como principales malestares físicos las dorsalgias, seguidas por la astenia, las cefaleas, los mareos y las abdominalgias. En relación con los casos de dismenorrea, los autores hallaron una realidad similar en su estudio, ya que un $20 \%$ de las mujeres participantes presentaba problemas en su menstruación. El no recibir atención médica refleja la poca asistencia a servicios de salud propia de esta población, como ya se ha mencionado lo cual, aunado con este tipo de síntomas que pasan desapercibidos, podría colocar a la persona adolescente en una posición de mayor vulnerabilidad.

Respecto del consumo de drogas, la Escuela de Salud Pública de la Universidad de Costa Rica (2010) establece que a nivel nacional las drogas legales son las más consumidas, en particular el alcohol, mientras que las drogas ilícitas atañen mayoritariamente a los adultos y no a los menores de 18 años. Del mismo modo, dicho ente plantea que a partir del año 1995 se ha observado un crecimiento sostenido en el consumo del licor en el caso de las mujeres, realidad presente en este estudio.

Las conductas encontradas para el área de la sexualidad en los participantes son acordes a la etapa de adolescencia media que experimentan, incluso, el Centro Nacional de Equidad de Género y Salud Reproductiva (2012) indica que los tocamientos en esta etapa de la vida son comunes y se realizan como una forma de exploración y aventura. Por otro lado, los resultados obtenidos en relación con el desarrollo puberal concuerdan con la máxima velocidad de crecimiento puberal esperada en el rango de 13 a 15 años (Shutt-Aine y Maddaleno, 2003).

El análisis de los factores protectores que permitió la categorización de los participantes, se elaboró con base en la subdivisión establecida por Zubarew (2013) a saber, factores concernientes al individuo, la familia y la comunidad. Específicamente, los factores de protección del segundo grupo son congruentes con las tareas propias del ciclo vital de "Conquistar la adolescencia" (Rodríguez-Araya, 2013).

Al analizar los datos referentes a las reacciones y comportamientos del grupo de participantes, recolectados a través de la hoja de vivencia, se corroboran las fases de la entrevista propuestas por Ruiz-Lázaro (2009), en la que, en un primer momento, la persona adolescente y el profesional mantienen una conversación trivial, lo que corresponde a la fase de acoplamiento, estrategia utilizada frente a la limitada expresión de sentimientos y/o pensamientos. Posteriormente se llega la fase exploratoria mediante el intercambio de información más detallada que permite identificar necesidades particulares, lo que alude al cambio de actitud de los adolescentes, lograda conforme avanza la sesión. De esta manera se alcanza la fase número tres o resolutiva en la que se formula los acuerdos y conclusiones, lo que equivale a la retroalimentación, clasificación según riesgo y el establecimiento de planes de acción en conjunto con la persona.

Entre las características esperadas en las investigadoras durante la implementación de las entrevistas, de acuerdo con González-Quirós (2010), es necesaria la escucha activa por parte del o la profesional ante la voz de 


\section{Revista Electrónica Enfermeria Actual en costa Rica}

este grupo etario, y tener claridad de las diferencias existentes entre cada uno de los adolescentes, su clase socioeconómica, origen cultural y familiar. Tal como lo plantea King (1984), las características y recursos implementados por la entrevistadora facilitaron que enfermería se acercara a la persona adolescente, para conocer la percepción que tienen de sí, así como sus necesidades y los acontecimientos que experimentan día a día dentro de su rol social.

En cuanto al abordaje grupal desarrollado por medio de la técnica de talleres, Ghiso (1999) expresa que esta estrategia favorece la socialización, la transferencia, la apropiación y el desarrollo de conocimientos, actitudes y competencias de una manera participativa y pertinente a las necesidades de los participantes. Lo anterior es importante en tanto que los adolescentes construyeron el conocimiento mediante su participación activa, el material informativo brindado y haciendo referencia con sus experiencias previa.

En relación con el taller de sexualidad, se evidenció un nivel básico de conocimiento sobre la temática, además de que hubo una apertura a recibir información clara y certera, a esclarecer mitos y a participar de forma activa en cada una de las actividades. Cabe destacar que a pesar de la gran influencia que ejerce la tecnología en la población adolescente (Echeburúa, 2010), la técnica del blog mostró ser un recurso poco familiar para esta población.

Con respecto al taller de prevención del consumo de drogas, los adolescentes se mostraron participativos, anuentes a discutir sus diferentes posiciones e interesados en la temática. Así, por ejemplo, en el tópico sobre el primer contacto con las sustancias, las frases mencionadas concuerdan con el discurso particular de esta problemática ya que la confianza referida es característica en el inicio del consumo de drogas. Al respecto el Instituto Costarricense sobre Drogas, ICD (s.f), establece que la invitación por lo general, se da por parte de un amigo, un familiar, compañero, u otra persona allegada.

Por último, la intención de la herramienta de debate fue cumplida, puesto que se generó espacios de desequilibrio entre sus esquemas y conocimientos previos, para someterlos al conflicto con el propósito de modificar sus concepciones y construir nuevos aprendizajes (Ministerio de Educación, 2007). Según los resultados y las reacciones conseguidas durante el conversatorio sobre el proceso de adicción. se evidenció la gran influencia de la presión social en la toma de decisiones (Instituto Costarricense sobre Drogas, s.f.).

Con base en los hallazgos de la evaluación final, particularmente en el taller de sexualidad segura, se destaca la costumbre de recibir información de forma magistral y poco interactiva. No obstante, da la impresión de que la expectativa acerca del taller de prevención de consumo de drogas fue mayor, vinculada con la experiencia previa de conocer la metodología constructivista-participativa.

Respecto de los cambios planteados para ambos talleres, sobresale la necesidad de hacer uso de un modelo anatómico que permitiera explicar la estructura interna de los órganos reproductores tanto masculinos como femeninos, esto debido a que suscitaron dudas al respecto. En cuanto a la recomendación brindada para el taller de prevención del consumo de drogas, se refleja la curiosidad característica de este periodo (Murillo-Castro, 2013), por lo que uno de sus intereses es obtener la mayor información posible sobre los efectos fisiológicos 


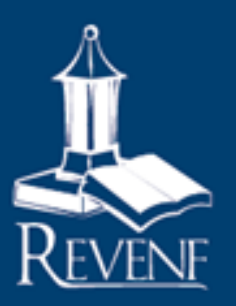

\section{Revista Electrónica Enfermeria Actual en costa Rica}

provocados por cada una de las sustancias y su duración en el organismo. Pese a ello, prevaleció el objetivo determinado por las investigadoras de brindar información orientada a la prevención del uso y abuso de las drogas.

Los rasgos de las facilitadoras para la estrategia grupal concuerdan con lo expresado por Krause (1995), quien afirma que el profesional debe fusionarse como entidad con la población para sumergirse en el discurso de los adolescentes, para poder "coconstuir" el conocimiento durante el programa. Dichos rasgos son necesarios en el perfil del profesional que atiende a este grupo etario (Gaete, 2011)

\section{CONCLUSIONES}

El criterio de los profesionales de enfermería pediátrica es indispensable para orientar el abordaje de las necesidades identificadas y la caracterización de factores de riesgo y/o protectores; por tanto, analizar de forma continua los hallazgos de un programa define el abordaje, el uso de metodologías afines y exige la articulación de varios actores sociales tales como educación, salud, organizaciones no gubernamentales, comunidad, familia, entre otros.

Las alteraciones más comunes en el grupo de adolescentes estuvieron relacionadas con los hábitos. Además, en su desarrollo, resultó importante las relaciones cotidianas con sus pares, en segunda instancia la importancia del apoyo familiar, las buenas relaciones en el colegio y la comunidad.

El programa facilitó y orientó la labor de los profesionales de enfermería pediátrica con la población adolescente, por lo que se convierte en una herramienta de trabajo clara, efectiva y acorde con la situación particular hallada.

Dentro de la atención es fundamental la intervención y el seguimiento de la persona adolescente, lo cual precisa promover factores protectores presentes como una herramienta para atenuar las necesidades (factores de riesgo) y fortalecer su capacidad para tomar control de su estado de salud.

\section{Conflicto de intereses}

Las autoras declaran no tener conflicto de intereses para la realización de esta investigación. 


\section{Revista Electrónica Enfermeria Actual en costa Rica}

\section{www.revenf.ucr.ac.cr}

\section{REFERENCIAS BIBLIOGRAFICAS}

Belamendia, M., Penzo, M., Bazano, M., Hortonella Y., Grunbaum, S., Durán P., Martínez, G., Maddaleno, M., De Mucio, B., y Fescina, R. (2010). Sistema Informático del Adolescente (SIA): historia clínica del adolescente y formularios complementarios: instrucciones de llenado y definición de términos. Montevideo: Centro Latinoamericano de Perinatología, Salud de la Mujer y Reproductiva.

Caja Costarricense del Seguro Social. (2012). Evaluación del desempeño de la prestación de servicios de salud. Informe de resultados 2011. San José: CCSS.

Centro Nacional de Equidad de Género y Salud Reproductiva. (2012). Sexualidad en la Adolescencia. México D.

F.: Secretaría de Salud.

Asamblea Legislativa de la República de Costa Rica. (2001). Código de la Niñez y la Adolescencia. Costa Rica.

Echeburúa, E. (2010). Adicción a las nuevas tecnologías y a las redes sociales en jóvenes: un nuevo reto. Adicciones , 22 (2), 91-96.

Escuela de Salud Pública UCR. (2010). La Salud Pública en Costa Rica: Estado actual, retos y perspectivas. San José: SIEDIN UCR.

Federación Española de Medicina del Deporte. (2008). La utilidad de la actividad física y de los hábitos adecuados de nutrición como medio de prevención de la obesidad en niños y adolescentes. Separata de Archivos de Medicina del Deporte , 25 (127), 333-353.

Fondo de las Naciones Unidas para la Infancia. (2011). Estado Mundial de la Infancia. La adolescencia una época de oportunidades. Nueva York: UNICEF.

Gaete, V. (2011). Atención clínica del adolescente que consulta por un problema de salud. Revista médica Clínica Las Condes , 22 (1), 5-13.

Ghiso, A. (1999). Acercamientos: el taller en procesos de investigación interactivos. Estudios sobre las Culturas Contemporáneas , 5 (9), 141-153.

González-Quirós, M. (2010). Análisis del aporte de la creación de la consulta de Enfermería Pediátrica para los niños, niñas y preadolescentes de los Centros Infantiles de la Asociación Roblealto en el año 2009. San José: Universidad de Costa Rica.

Grupo Avena. (2003). Alimentación y valoración del estado nutricional de los adolescentes españoles: Evaluación de riesgos y propuesta de intervención. Madrid: Nutrición Hospitalaria XVIII. 


\section{Revista Electrónica Enfermeria Actual en costa Rica}

Hernández-Sampieri, R., Fernández-Collado, C., y Baptista-Lucio, P. (2010). Metodología de investigación (5ta ed.). México D.F.: McGraw-Hill.

Instituto Costarricense sobre Drogas. (s.f.). Drogas, preguntas más frecuentes. Recuperado de: http://www.icd.go.cr/portalicd/images/docs/upp/publicacionesUPP/Drogas_preguntas_m\%C3\%A1s_frecue ntes_ld.pdf

Instituto Costarricense sobre Drogas. (2014). Saber elegir, saber ganar. San José, ICD. Recuperado de: http://www.icd.go.cr/portalicd/index.php/programas-upp-delito/saber-elegir

Instituto Nacional de Estadística y Censos. (2011). Costa Rica: Población total por grupos de edad, según provincia, cantón y sexo. Recuperado de http://www.inec.go.cr/Web/Home/GeneradorPagina.aspx

King, I. (1984). Enfermería como profesión. Filosofía, principios y objetivos. México, D.F.: LIMUSA.

Krause, M. (1995). La investigación cualitativa. Un campo de posibilidades y desafíos. Temas de Educación (7), $1-18$.

Llusent-Guillamet, A., Agüero-Martínez, N., y Cornellà-Canals, J. (2007). La percepción de "salud" en adolescentes supuestamente sanos. Programa "Salut i Escola" Girona:analesdepediatría.

Marugán de Miguelsanz, J., Monasterio-Corral, L., y Pavón-Belinchón, M. (2010). Alimentación en el adolescente. En H. y Asociación Española de Pediatría-Sociedad Española de Gastroenterología, Protocolos diagnóstico-terapéuticos de Gastroenterología, Hepatología y Nutrición Pediátrica. Santiango de Compostola: ERGON.

Ministerio de Educación. (2007). Corrientes Pedagógicas y Psicológicas que influyen en la formación de púberes y adolescentes. Lima: Ministerio de Educación de la República del Perú.

Murillo-Castro, L. (2013). El policonsumo de las drogas ilícitas en los adolescentes de Hogares CREA de Barva de Heredia y Cartago. Revista Enfermería Actual en Costa Rica (24), 1-13.

Organización de Naciones Unidas. (1989). Convención sobre los derechos del niño. Recuperado de: http://www.margen.org/ninos/derecho8.html

Organización de las Naciones Unidas. (2007). Oficina del Alto Comisionado de las Naciones Unidas para los Derechos Humanos. Recuperado de: http://www2.ohchr.org/spanish/law/crc.htm 


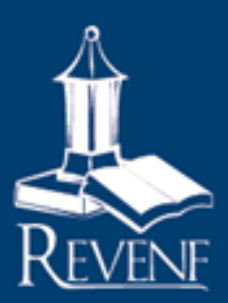

\section{Revista Electrónica Enfermeria Actual en costa Rica}

Organización Panamericana de la Salud. (2010). Propuesta de Revisión del Módulo de Atención de los y las adolescentes en el marco de la integración del manejo adolescente y sus necesidades (IMAN) en el nivel primario de atención. OPS.

PANI- UNICEF. (2009). Política Nacional para la Niñez y la Adolescencia Costa Rica 2009-2021. Costa Rica.

Pin-Arboledas, G. (2010). Bases fisiológicas y anatómicas del sueño. Evolución del sueño en la infancia y adolescencia. Clasificación internacional de los trastornos del sueño. Hábitos de sueño de la población española. Pediatría Integral , 14 (9), 691-698.

Quevedo-Blasco, V. y Quevedo-Blasco, R. (2011). Influencia del grado de somnolencia, cantidad y calidad de sueño sobre el rendimiento académico en adolescentes. International Journal of Clinical and Health Psychology , 11 (1), 49-65.

Retana-Goñi, M. (2007). Formas en que se puede manifestar y reproducir conductas violentas hacia la pareja en las relaciones de noviazgo. San José: Universidad de Costa Rica.

Rodríguez-Araya, A. (2013). Unidad II: La familia como unidad vital de apoyo y contención en el contexto social. En G. Casas, Qué se diagnostica en un sistema familiar. San José: Universidad de Costa Rica.

Ruiz-Lázaro, P. (2009). La entrevista clínica al adolescente. Desarrollo Profesional , 31-33.

Shutt-Aine, J., y Maddaleno, M. (2003). Salud sexual y desarrollo de adolescentes y jóvenes en las Américas: Implicaciones en programas y políticas. Washington, DC: Organización Panamericana de la Salud.

Vidal-Ledo, M., y Rivera-Michelena, N. (2007). Investigación-Acción. Educación Médica Superior, 21(4), 1-15.

Zubarew, T. (2013). Evaluación Clínica del Adolescente. Supervisión de Salud del Adolescente. Lección 2. parte 1. Santiago: Pontificia Universidad Católica de C 